\title{
Coupled Resonator Diplexer without External Junctions
}

\section{Talal Skaik, Michael Lancaster}

School of Electronic, Electrical and Computer Engineering, University of Birmingham, Birmingham, U.K. Email: talalskaik@gmail.com

Received April $5^{\text {th }}, 2011$; revised May $9^{\text {th }}, 2011$; accepted May $17^{\text {th }}, 2011$.

\begin{abstract}
A coupled resonator diplexer has been designed, fabricated, and tested. The design is based on synthesis of coupling matrix of a 3-port coupled resonator circuit using optimization. Unlike conventional diplexers, the presented diplexer configuration does not include any separate junctions for distribution of energy. The diplexer has been implemented at $X$-band with waveguide cavity resonators, and its measured response is in good agreement with the simulated response.
\end{abstract}

Keywords: Coupled-Resonator, Coupling Matrix, Diplexer, Optimization

\section{Introduction}

Microwave diplexers are widely used in communication systems to connect two networks operating at different frequencies to a common port. Conventional diplexers consist of two channel filters connected to an energy distribution network. Such a network can be a waveguide manifold [1], a T-junction [2], a Y-junction [3], or a circulator [4]. A common approach for the diplexer synthesis is based on firstly designing the channel filters independently of the diplexer, and then using numerical optimization for the whole diplexer structure. This approach can be very time consuming for large diplexer structures and the convergence of the cost function might be problematic. In $[5,6]$, an approach to the synthesis of diplexers that takes into account a three-port junction in the initial synthesis of the two channel filters was presented. This approach provides a very good starting point for the optimization of the whole structure, so the convergence can be achieved with few iterations. Diplexers/Multiplexers employing resonant junctions (an extra resonator in addition to the resonators of the channel filters) have also been reported in [5,7].

Multiplexers/diplexers based on coupled resonator circuits with multiple outputs have been reported in [8-11]. In [8], coupled resonator circuits with multiple outputs were first patented. In $[9,10]$, the synthesis of multiport coupled resonator networks based on a procedure analogous to filters has been reported. The proposed configurations in these papers do not employ any external junctions for distribution of energy, and hence, the components can be miniaturized. The diplexer proposed in [9], however, may present difficulties in implementation, since the common port is directly coupled to two resonators.

In [12], we presented a design procedure for coupled resonator diplexers that do not employ any external junctions. The proposed diplexers have novel topologies that can be implemented by any type of resonators, and their synthesis is based on coupling matrix optimization. In this paper, we present a coupled resonator diplexer that has been synthesized using coupling matrix optimization. The diplexer has non-contiguous bands with symmetrical channels, and it is implemented at X-band using twelve waveguide cavity resonators to verify our design approach in [12]. The proposed diplexer is miniaturized when compared to the conventional diplexers, since it does not contain external junctions. In addition, miniaturization is improved by using a folded structure.

\section{Diplexer Synthesis}

The synthesis is based on optimization of the coupling matrix for multiple coupled resonators arranged in a 3 -port network. A general matrix $[A]$, derived for a multiport coupled-resonator circuit, in terms of the coupling coefficients and the external quality factors is given in (1) $[12,13]$, where $q_{e i}$ is the normalized external quality factor of resonator $i, P$ is the complex lowpass frequency variable, $m_{i j}$ is the Normalized coupling coefficient between resonators $i$ and $j$, and the diagonal entries $m_{i i}$ account for asynchronous tuning. 


$$
\begin{aligned}
{[A] } & =\left[\begin{array}{cccc}
1 / q_{e 1} & \cdots & 0 & 0 \\
\vdots & \vdots & \vdots & \vdots \\
0 & \cdots & 1 / q_{e(n-1)} & 0 \\
0 & \cdots & 0 & 1 / q_{e n}
\end{array}\right]+P\left[\begin{array}{cccc}
1 & \cdots & 0 & 0 \\
\vdots & \vdots & \vdots & \vdots \\
0 & \cdots & 1 & 0 \\
0 & \cdots & 0 & 1
\end{array}\right] \\
& -j\left[\begin{array}{cccc}
m_{11} & \cdots & m_{1(n-1)} & m_{1 n} \\
\vdots & \vdots & \vdots & \vdots \\
m_{(n-1) 1} & \cdots & m_{(n-1)(n-1)} & m_{(n-1) n} \\
m_{n 1} & \cdots & m_{n(n-1)} & m_{n n}
\end{array}\right]
\end{aligned}
$$

Considering a 3-port coupled-resonator network, and assuming that port 1 is coupled to resonator 1 , port 2 is coupled to resonator $a$ and port 3 is coupled to resonator $b$, the scattering parameters are related to the matrix $[A]$ by [12]:

$$
\begin{gathered}
S_{11}=1-\frac{2}{q_{e 1}}[A]_{11}^{-1} \\
S_{21}=\frac{2}{\sqrt{q_{e 1} q_{e a}}}[A]_{a 1}^{-1}, \quad S_{31}=\frac{2}{\sqrt{q_{e 1} q_{e b}}}[A]_{b 1}^{-1}
\end{gathered}
$$

The optimization of the coupling matrix $[\mathrm{m}]$ is based on minimization of a cost function that is evaluated at the frequency locations of the reflection and transmission zeros. The cost function used here is given as [12],

$$
\begin{aligned}
\Omega & =\sum_{i=1}^{T_{1}}\left|\frac{2}{\sqrt{q_{e 1} q_{e a}}} \cdot \operatorname{cof}_{1 a}\left(\left[A\left(s_{t i}\right)\right]\right)\right|^{2} \\
& +\sum_{k=1}^{T_{2}}\left|\frac{2}{\sqrt{q_{e 1} q_{e b}}} \cdot \operatorname{cof}_{1 b}\left(\left[A\left(s_{t k}\right)\right]\right)\right|^{2} \\
& +\sum_{j=1}^{R}\left|\Delta_{A}\left(s_{r j}\right)-\frac{2 \cdot \operatorname{cof}_{11}\left(\left[A\left(s_{r j}\right)\right]\right)}{q_{e 1}}\right|^{2} \\
& +\sum_{v=1}^{R-2}|| \frac{2 \cdot \operatorname{cof}_{11}\left(\left[A\left(s_{p v}\right)\right]\right)}{q_{e 1} \cdot \Delta_{A}\left(s_{p v}\right)}\left|-10^{\frac{L_{R}}{20}}\right|^{2}
\end{aligned}
$$

where $\operatorname{cof}_{k h}([A(s=x)])$ is the cofactor of matrix $[A]$ evaluated by removing the $k$-row and the $h$-column of $[A]$ and calculating the determinant of the resulting matrix at $s=x . \Delta_{A}$ is the determinant of the matrix $[A] . s_{t i}, s_{t k}$ are the frequency locations of transmission zeros of $S_{21}, S_{31}$ respectively. $T_{1}, T_{2}$ are the numbers of the transmission zeros of $S_{21}, S_{31}$ respectively, and $R$ is the total number of resonators. $L_{R}$ is the specified return loss in $\mathrm{dB}\left(L_{R}<0\right)$, and $s_{r j}$ and $s_{p v}$ are the frequency locations of the return zeros and the peaks' frequency values of $\left|S_{11}\right|$ in the passband. The last term in the cost function is used to set $\left|S_{11}\right|$ peaks to the specified value of $L_{R}$.

\section{Diplexer Design}

An X-band 12-resonator non-contiguous diplexer with symmetrical channels has been designed and implemented using waveguide cavity resonators. The diplexer has a Chebyshev response with passband centre frequency of $10 \mathrm{GHz}$ for channel 1 and $11.35 \mathrm{GHz}$ for channel 2, minimum isolation of $60 \mathrm{~dB}$, and a desired return loss at the passband of each channel is $20 \mathrm{~dB}$. The diplexer topology is shown in Figure 1. The proposed diplexer is formed of only coupled resonators, without using any external junctions for energy distribution, and the structure is folded for miniaturization. A gradient based constrained optimization technique has been utilized to synthesize the coupling coefficients, and the cost function in equation (3) has been used. To avoid convergence to a local minimum, the optimization has been carried out in two stages. In the first stage, the cost function in (3) has been used without the last term, and with equally spaced reflection zeros. In the second stage, the full cost function in (3) has been used, and the output coupling coefficients from the first stage are used as initial values, and the frequency locations of the return zeros are allowed to move until $\left|S_{11}\right|$ peaks in the passbands are equal to $-20 \mathrm{~dB}$. Moreover, the third term in the cost function was assigned more weight than the other terms. Although a Chebyshev response is required, the first two terms in the cost function have been used to increase the isolation between diplexer channels by minimizing the transmission of each channel at the passband of the other channel.

The optimized normalized coupling coefficients are as follows: $m_{12}=0.7963, m_{23}=m_{28}=0.3466, m_{34}=m_{89}=$ $0.2101, m_{45}=m_{9,10}=0.195, m_{56}=m_{10,11}=0.2035, m_{67}=$ $m_{11,12}=0.2814, m_{33}=-m_{88}=0.5942, m_{44}=-m_{99}=0.655$, $m_{55}=-m_{10,10}=0.6635, m_{66}=-m_{11,11}=0.6652, m_{77}=$ $-m_{12,12}=0.6643$. The fractional bandwidth is $F B W=$ $18 \%$, and the normalized external quality factors are numerically calculated as in [12] and found to be $q_{e 1}=$ 1.4903 and $q_{e 7}=q_{e 12}=2.9806$. The prototype response of the diplexer is shown in Figure 2.

The diplexer has been implemented using inductively coupled waveguide cavity resonators, and a top view of diplexer structure is shown in Figure 3.

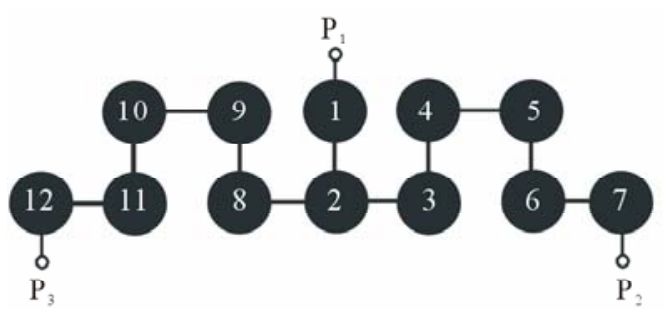

Figure 1. Diplexer topology. 


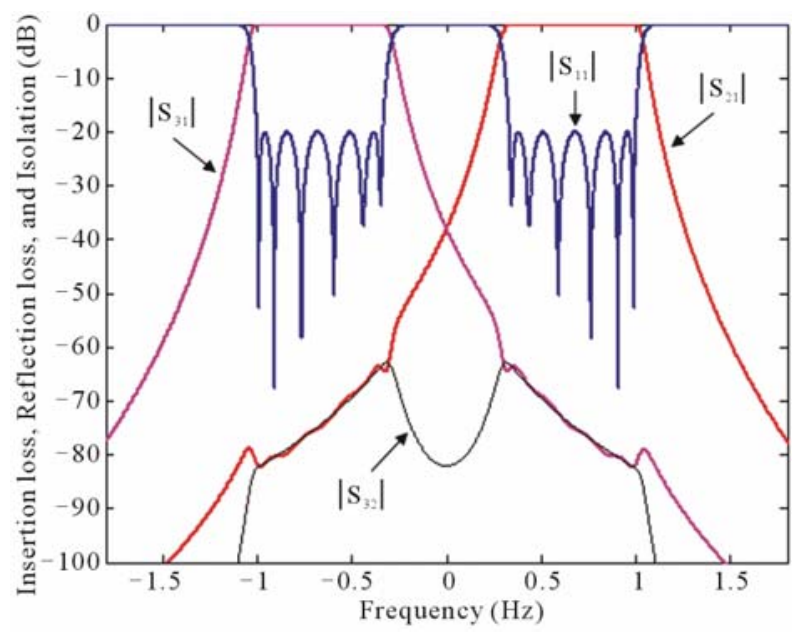

Figure 2. Diplexer prototype response.

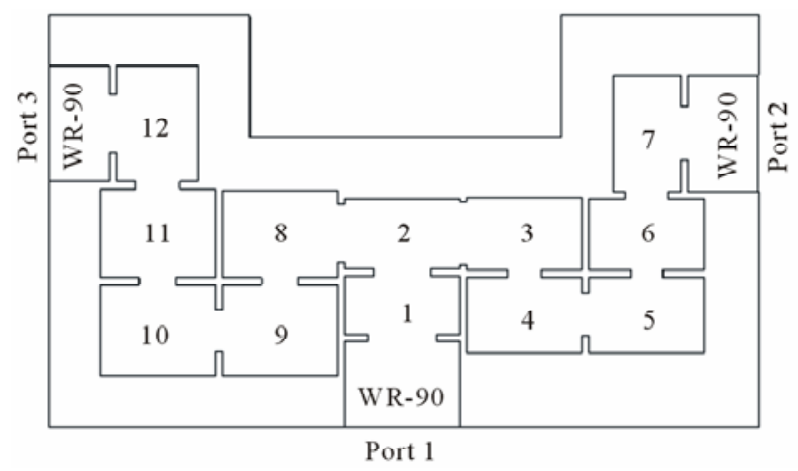

Figure 3. Top view of diplexer structure.

\section{Fabrication and Measurement}

The diplexer has been made of two mirror-image pieces of copper, and a photograph of the physical structure of the diplexer is shown in Figure 4. Metal screws have been used to tune both the resonant frequencies of the cavities and the coupling coefficients. The simulated and measured results of the diplexer are depicted in Figure 5. The tuned measured response is in very good agreement with the simulated response. The measurements show that the passband of channel 1 has maximum return loss of $\sim 18 \mathrm{~dB}$ and minimum insertion loss of $0.42 \mathrm{~dB}$, and that the passband of channel 2 has maximum return loss of $22 \mathrm{~dB}$ and minimum insertion loss of $0.4 \mathrm{~dB}$.

\section{Conclusions}

An X-band coupled resonator diplexer has been presented, and its synthesis is based on coupling matrix optimization. The diplexer structure consists of resonators coupled together, and it does not involve any external junctions for distribution of energy. This enables miniaturization in comparison to the conventional diplexers. The diplexer has been implemented with waveguide cavity

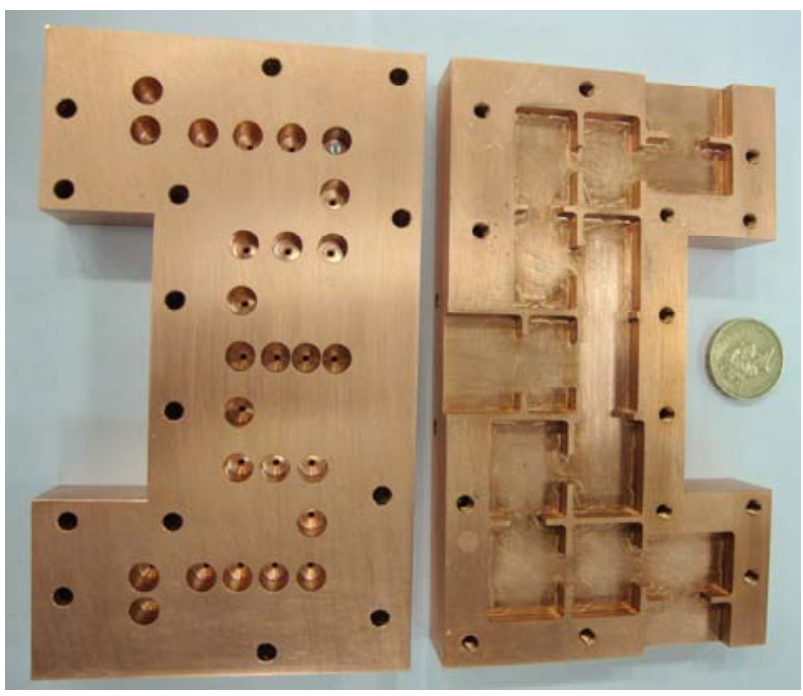

Figure 4. Photograph of the diplexer.

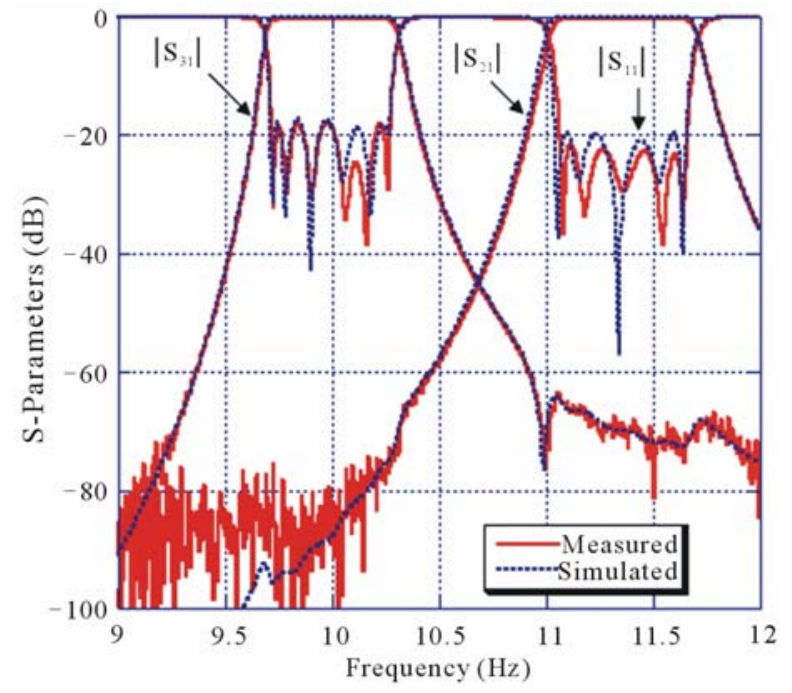

Figure 5. Measured and simulated results of the diplexer.

resonators, and the measured results showed a good agreement with the simulated results.

\section{REFERENCES}

[1] M. Guglielmi, "Optimum CAD Procedure for Manifold Diplexers," Proceeding of IEEE MTT-S International Microwave Symposium Digest, Atlanta, 14-18 June 1993, pp. 1081-1084. doi:10.1109/MWSYM.1993.277054

[2] H. Zhang and G. James, "A Broadband T-junction Diplexer with Integrated Iris Filters," Microwave and Optical Technology letters, Vol. 17, No. 1, 1998, pp. 69-72. doi:10.1002/(SICI)1098-2760(199801)17:1<69::AID-MO P19>3.0.CO;2-6

[3] S. Bastioli, L. Marcaccioli and R. SorrentiNo, "An Original Resonant Y-junction for Compact Waveguide Diplexers," Proceeding of IEEE MTT-S International 
Microwave Symposium Digest, Boston, 7-12 June 2009, pp. 1233-1236. doi:10.1109/MWSYM.2009.5165926

[4] C. E. Saavedra, "Diplexer Using a Circulator and Interchangeable Filters," Proceeding of the 7th International Caribbean Conference on Devices, Circuits and Systems, Mexico, 28-30 April 2008, pp. 1-5.

doi:10.1109/ICCDCS.2008.4542631

[5] G. Macchiarella and S. Tamiazzo, "Novel Approach to the Synthesis of Microwave Diplexers," IEEE Transactions on Microwave Theory and Techniques, Vol. 54, No. 12, 2006, pp. 4281-4290. doi:10.1109/TMTT.2006.885909

[6] G. Macchiarella and S. Tamiazzo, "Synthesis of Diplexers Based on the Evaluation of Suitable Characteristic Polynomials," IEEE MTT-S International Microwave Symposium, San Francisco, 11-16 June 2006, pp. 111-114. doi:10.1109/MWSYM.2006.249406

[7] G. Macchiarella and S. Tamiazzo, "Synthesis of StarJunction Multiplexers," IEEE Transactions on Microwave Theory and Techniques, Vol. 58, No. 12, 2010, pp. 3732-3741. doi:10.1109/TMTT.2010.2086570

[8] M. J. Lancaster, "Radio Frequency Filter," World Intellectual Property Organization Patent, WO/01/69712, 2001.

[9] A. Garcia-Lamperez, M. Salazar-Palma and T. K. Sarkar,
"Analytical Synthesis of Microwave Multiport Networks," IEEE MTT-S International Microwave Symposium Digest, 6-11 June 2004, pp. 455-458. doi:10.1109/MWSYM.2004.1336009

[10] A. Garcia-Lamperez, M. Salazar-Palma, and T. K. Sarkar, "Compact Multiplexer Formed by Coupled Resonators with Distributed Coupling," IEEE Antennas and Propagation Society International Symposium, 3-5 July 2005, pp. 89-92. doi:10.1109/APS.2005.1551250

[11] F. Loras-Gonzalez, S. SobriNo-Arias, I. Hidalgo-Carpintero, A. Garcia-Lamperez and M. Salazar-Palma, "A Novel $\mathrm{Ku}$-band Dielectric Resonator Triplexer Based on Generalized Multiplexer Theory," IEEE MTT-S International Microwave Symposium Digest, Anaheim, 23-28 May 2010, pp. 884-887. doi:10.1109/MWSYM.2010.5515905

[12] T. Skaik, M. Lancaster and F. Huang, "Synthesis of Multiple Output Coupled Resonator Microwave Circuits Using Coupling Matrix Optimization," IET Journal of Microwaves, Antennas and Propagation, Vol. 5, No. 9, 2011. pp. 1081-1088. doi:10.1049/iet-map.2010.0447

[13] T. Skaik, M. Lancaster and F. Huang, "Coupled-Resonator 3-dB Power Divider," Proceedings of the IET seminar on Passive RF and Microwave Components, Birmingham, April 2010, pp. 21-36. 